\title{
The Five-Parameter Grain Boundary Curvature Distribution in an Austenitic and Ferritic Steel
}

Xiaoting Zhong, ${ }^{1}$ David J. Rowenhorst, ${ }^{2}$ Hossein Beladi, ${ }^{3}$ and Gregory S. Rohrer ${ }^{1,}{ }^{*}$

\begin{abstract}
The distribution of grain boundary curvatures as a function of five independent crystallographic parameters is measured in an austenitic and a ferritic steel. Both local curvatures and integral mean curvatures are measured from three dimensional electron backscattered diffraction data. The method is first validated on ideal shapes. When applied to real microstructures, it is found that the grain boundary mean curvature varies with the boundary crystallography and is more sensitive to the grain boundary plane orientation than to the disorientation. The grain boundaries with the smallest curvatures also have low grain boundary energy and large relative areas. The results also show that the curvature is influenced by the grain size and by the number of nearest neighbors. For austenite, when the number of faces on a grain is equal to the average number of faces of its neighbors, it has zero integral mean curvature.
\end{abstract}

Keywords: 3D microstructure, grain boundary curvature, grain boundaries, microstructure 


\section{Introduction}

Recent advances in three-dimensional (3D) materials characterization methods have made it possible to collect the first large sets of data on grain shape, [1,2] grain topology, [3] grain boundary populations, [4] the evolution of the grain boundary population, [5] and grain boundary energies. [6] Grain boundary curvature is one of the most important properties of a microstructure because the product of the curvature and energy provide the driving force for grain growth. Using measurements from about $2000 \beta$-Ti grains, Rowenhorst et al. [3] found that grains with 15 or fewer faces have, on average, a positive integral mean curvature and grains with 16 or more faces have, on average, a negative integral mean curvature. Although the integral mean grain boundary curvature is known to be correlated to the grain size and the number of grain faces [3], less is known about its correlation to grain boundary crystallography.

Grain boundaries have five independent crystallographic parameters; three describe the lattice misorientation $(\Delta g)$ and two describe the grain boundary plane orientation (n). [7] While studies of lattice misorientations have a long history, it has only been through 3D studies that direct measurements of the grain boundary plane orientations have been possible. Grain boundary properties, and the macroscopic properties of polycrystals, can be sensitive to grain boundary plane orientations. [8-10] Therefore, the characterization of all five parameters can be important for establishing structure-property relations for polycrystalline materials. A number of measurements have been reported of grain boundary populations and grain boundary energies as a function of all five parameters. [11-15] One general conclusion is that in microstructures formed by normal grain growth, the grain boundary population is inversely correlated to the grain boundary energy. [16]

Zhong et al. p. 2 
It has not yet been established whether a relationship exists between local grain boundary curvature and the local grain boundary crystallography. However, based on anecdotal observations of microstructure, there is support for a correlation. For example, the coherent twin boundary in FCC metals, which has a well-defined crystallography, is often very flat (it has minimal curvature). The coherent twin boundary also has minimal energy and a relative large area fraction [12].

Grain growth theories usually assume that, at any instance in time, there is a constant mean field chemical potential in the microstructure and that local deviations from this mean value drive grain growth. $[17,18]$ For example, in Hillert's $[17,18]$ classic grain growth theory, there is a critical radius $\left(r_{c r}\right)$ above which grains grow and below which they shrink; the time rate of change of a grain with radius $r$ is then proportional to the difference between the chemical potential of a grain with radius and $r$ and one with radius $r_{c r}$, which has the mean field chemical potential. In Hillert's formulation, the chemical potential is not dependent the grain boundary crystallography. However, the chemical potential in its most general form does depend on the grain boundary crystallography. This is clear from the way Herring [19-21] expressed the chemical potential $\left(\mu_{n s}\right)$ of a nonsingular surface at a specific point in equilibrium:

$\mu_{n s}=\left(\gamma(\Delta g, \mathbf{n}),+\frac{\partial^{2} \gamma(\Delta g, \mathbf{n})}{\partial \theta_{1}^{2}}\right) \kappa_{1}+\left(\gamma(\Delta g, \mathbf{n})+\frac{\partial^{2} \gamma(\Delta g, \mathbf{n})}{\partial \theta_{2}^{2}}\right) \kappa_{2}$

where $\chi \Delta g, \mathbf{n})$ is the grain boundary energy, $\kappa_{1}$ and $\kappa_{2}$ are the principal curvatures, $\theta_{1}$ is the angle between the normal to the grain boundary at the point of interest and another normal at a nearby point on the boundary that is also in the plane of principal curvature 
corresponding to $\kappa_{1}$ and $\theta_{2}$ is the corresponding quantity for the perpendicular plane. In other words, the derivative terms in Eqn. 1 characterize variations of the grain boundary energy within the planes of principal curvature. If we ignore the second derivatives for the moment and define the mean curvature as $H(\Delta g, \mathbf{n})=1 / 2\left(\kappa_{1}+\kappa_{2}\right)$, then Eq. 1 reduces to the simplified form:

$$
\mu_{n s}=2 H(\Delta g, \mathbf{n}) \gamma(\Delta g, \mathbf{n})
$$

Assuming a constant mean field chemical potential, Eqn. 2 argues for an inverse relationship between the mean curvature and the grain boundary energy. Because the grain boundary energy varies with grain boundary crystallography, the mean curvature should also depend on the grain boundary crystallography. Grain boundary energies have been measured based on the assumption of local equilibrium at triple junctions [6, 11-15] and the measurements produce results that are consistent with calculations, at least of the most common, lowest energy grain boundaries. [22] Unfortunately, the energies of grain boundaries that occur infrequently are not well-determined and, because of this, the second derivatives of the measured grain boundary energies needed to apply Eqn. 1 are not likely to be meaningful.

The argument above applies to grain boundaries with non-singular orientations, which are orientations where the grain boundary energy versus orientation at fixed disorientation, $\chi(\Delta g \mid \mathbf{n})$, is continuous and differentiable. The same will not be true for singular orientations, which occur at cusps in $\chi(\Delta g \mid \mathbf{n})$. [23] The chemical potential of a singular grain boundary is proportional to its weighted mean curvature (which is inversely

Zhong et al. p. 4 
proportional to the size of the grain and is comparable to curvature of a non-singular boundary) [24] multiplied by the area weighted average energy of the boundaries that are on the periphery of the singular interface, $\left\langle\gamma\left(\Delta g \mid \mathbf{n}_{p}\right)>\right.$. [19] In other words, it is not the energy of the singular interface that determines its chemical potential, it is the energies of the boundaries that are nearest to the singular plane in orientation space. [19, 20,24] The reason for this is that the energy change that occurs when a flat facet advances (retracts) is proportional to the energy required to extend (shrink) the boundaries on the periphery of the facet. The exact form of the chemical potential of a singular boundary depends on its exact geometry and the details can be found in references $[19,20,24]$. We can write an approximate expression for the chemical potential of a singular boundary, $\mu_{s}$, that is analogous to Eqn. 2:

$$
\mu_{s} \approx \alpha \cdot<\gamma\left(\Delta g \mid \mathbf{n}_{p}\right)>
$$

Where $\alpha$ is the weighted mean curvature and includes geometric terms that are specific for each singular interface; the expression ignores a variable but small contribution from the singular boundary itself. While Eqn. 3 is highly approximate, it illustrates the most important difference between the chemical potential of a singular and non-singular boundary: the chemical potential of the non-singular boundary is proportional to the energy of that boundary while the chemical potential of a singular grain boundary depends on the energies of the grain boundaries that are peripheral to the singular orientation. In every case where there is more than one stable orientation, the peripheral orientations have higher energies than the singular orientation. [23]

Zhong et al. p. 5 
Based on what is known about the chemical potentials of singular and non-singular grain boundaries, and the assumption of a constant average chemical potential, we expect the correlation between grain boundary energy and curvature to be different for different types of boundaries. Singular grain boundary orientations should have low curvatures and low energies. This is consistent with the observation that twin boundaries are flat and have low energies. For non-singular grain boundaries, on the other hand, low energy boundaries are expected to have relatively higher curvatures.

The purpose of this paper is to test the hypothesis that the mean grain boundary curvature depends on the grain boundary crystallography. We test the hypothesis through a new analysis of 3D microstructure data from an austenitic steel and a ferritic steel for which the five-parameter grain boundary area and energy distributions have already been measured. $[14,15]$ Before analyzing the real microstructures, the accuracy of the grain boundary curvature measurement is tested on ideal shapes to establish how the uncertainty varies with the resolution and reconstruction procedures. We then determine how the integral mean curvature of the grains varies with the number of grain faces and with the average number of faces of its nearest neighbors. Finally, we compute the fiveparameter grain boundary curvature distribution for austenite and ferrite and compare it to the measured grain boundary energy.

\section{Procedures}

In this paper, we analyzed 3D orientation maps previously reported for two steels. The first is an austenitic twinning-induced plasticity (TWIP) steel with the face-centered cubic (FCC) structure and the second is a ferritic steel with the body-centered cubic (BCC) structure. Throughout this paper, these samples will simply be referred to as the austenitic 
and ferritic steels. The composition of the austenitic steel was $0.6 \mathrm{C}-18 \mathrm{Mn}-1.5 \mathrm{Al}$ (wt.\%) and the composition of the ferritic steel was $0.04 \mathrm{C}-1.52 \mathrm{Mn}-0.2 \mathrm{Si}-0.22 \mathrm{Mo}-0.08 \mathrm{Ti}-0.033$ $\mathrm{Al}$ (wt.\%). The orientation map of the austenitic steel was $65 \times 40 \times 20 \mu \mathrm{m}^{3}$ and contained 3185 grains. There were two orientation maps for the ferritic steel. One was $40 \times 35 \times 14$ $\mu \mathrm{m}^{3}$ and contained 1113 grains and the other was $30 \times 50 \times 22 \mu \mathrm{m}^{3}$ and contained 558 grains. Further details of these samples, including the processing used to produce these microstructures, have already been reported. $[14,15]$

The 3D orientation maps were constructed from stacks of parallel two-dimensional (2D) orientation maps obtained by electron backscatter diffraction (EBSD). The serial sectioning was carried out in a dual beam focused ion beam scanning electron microscope (FEI Quanta 3D FEGSEM). The raw data are available at the grain boundary data archive. [25] Open source software, DREAM.3D, [26] was then used to reconstruct the 3D orientation maps. After reading in the data, voxels with low image quality $(\leq 120)$ or confidence index $(\leq 0.1)$ were ignored. Image quality and confidence index are metrics that indicate the quality of the diffraction pattern and the confidence in the orientation assignment, respectively, that are assigned by the TSL OIM software (EDAX, Mahwah, NJ) that is used to collect the data. [27] Next, centroid and misorientation based algorithms were used to correct sub-pixel misalignment between the 2D sections. To segment the volume into grains, the orientation of each voxel was compared to its neighbors and voxels with orientations that differed by $\leq 5^{\circ}$ were grouped together and assigned a unique grain identification (ID) number. Furthermore, grains had to have at least 100 voxels and at least two neighboring grains. After this process, the microstructure contained gaps created by groups with fewer than 100 voxels, mostly made up of voxels with unknown orientations.

$\begin{array}{ll}\text { Zhong et al. p. } 7 & \text { p }\end{array}$ 
The gaps were eliminated by dilating neighboring grains uniformly using a procedure in DREAM.3D. [28, 29, 26] In the final step, all of the voxels within the grain were assigned the average orientation of all of the voxels in the group. The voxels that make up the data create stair-stepped boundaries and triple lines that must be smoothed to extract grain boundary plane distributions and grain boundary curvature distributions. While a method has recently been developed to compute average face curvatures from voxelated microstructures [30], we are interested in local curvatures and thus must represent the boundary as a surface composed of discrete triangles. A multi-material marching cube meshing algorithm was used to model the grain boundaries as triangular nets and a restricted Laplacian smoothing was used to create smoothly curved grain boundaries. [3] The details of the parameters used for the smoothing procedure and their effect on the results are considered in $§ 3.1$. At this stage, the reconstruction is complete and the microstructures are composed of discrete grains with unique ID numbers, bounded by a triangular mesh. Associated with each triangle in the mesh are the grain ID numbers on each side, the orientations of the grains on each side, the disorientation across the triangle, the surface normal, the area, and the curvature (the calculation of the curvature is described in the next paragraph). This makes it possible to map these quantities locally and to define how they are distributed over the crystallographic, volumetric, and topological parameters.

Grain boundary (mean) curvature was calculated locally for the triangles using the previously established cubic-order algorithm described in reference [31] and implemented in DREAM.3D. $[3,26]$ In this algorithm, a patch of contiguous triangles surrounding the triangle of interest, for which we know the crystallographic properties, is fit to a parabola.

$\begin{array}{ll}\text { Zhong et al. } & \text { p. } 8\end{array}$ 
The size of neighborhood patch used in our calculation includes $2^{\text {nd }}$ and $3^{\text {rd }}$ nearest neighbors of the center triangle. After using a least-squares procedure to fit the parabola, the Weingarten matrix of this surface patch can be obtained and its eigenvalues are the principal curvatures $\kappa_{1}$ and $\kappa_{2}$. Triangle mean curvature is then $\frac{\kappa_{1}+\kappa_{2}}{2}$ and its sign is defined as positive for convex and negative for concave. However, note that only the absolute values of the triangle mean curvatures were used in calculations of the grain boundary curvature distribution as a function of crystallographic parameters. The reason lies in the inversion symmetry between neighboring grains. While convex and concave are explicit for an individual grain, there is no way to define such a property for the network of boundaries between grains. After calculating the curvature value, each triangle was then classified in a discrete distribution according to its crystallographic parameters using the same methods that have been used to compute the grain boundary character and energy distributions. $[32,6,33]$ Briefly, the five grain boundary parameters are the three Euler angles $\left(\varphi_{1}, \boldsymbol{\Phi}, \varphi_{2}\right)$, specifying the lattice misorientation, and the two spherical angles, $(\Theta$, $\varphi$ ), specifying boundary normal direction. Because of crystal symmetries, there are many indistinguishable representations of each boundary in the complete domain of boundary types. Here, we classify the angles $\varphi_{1}, \boldsymbol{\Phi}, \varphi_{2}$ into discrete bins within the range from 0 to $90^{\circ}$. Similarly, we classify the spherical angle $\Theta, \varphi$ into discrete bins within the range from 0 to $90^{\circ}$ and 0 to $360^{\circ}$, respectively. [7] The discrete bins have equal volume and there are 9 bins per $90^{\circ}$. The five parameters of every triangle were examined and its triangle curvature value was added to the corresponding bin. Because of cubic crystal symmetry, there are 36 physically indistinguishable representations or combinations of angles for each triangle within the range of angles considered. [7] After applying the symmetry

Zhong et al. p. 9 
operators, the curvature was also added to all bins corresponding to indistinguishable representations. After all of the triangles were binned, the curvature values in each bin were averaged and this gives us the symmetry averaged curvature for the grain boundary curvature distribution (GBCurD).

\section{Results}

\subsection{Curvature measurements of spheres}

It should be recognized that curvatures measured from discrete voxelized data are necessarily approximations of the true grain boundary curvature. Intuitively, the accuracy of this approximation should depend on the resolution of the data (defined as the size of a voxel compared the size of a grain) and the way that the triangular mesh representing the grain boundary is smoothed. Furthermore, when the curvature of an individual triangle is classified in the discrete five-parameter distribution, it will be averaged with boundaries that have similar parameters. Therefore, the symmetry averaged curvatures will also be affected by the discrete nature of the distribution. To understand how these factors influence the curvature measurement, we examined the effect of the data processing on the measured curvatures of simulated spherical grains.

Ten spheres were created and labeled with different resolutions. For example, S4 denotes a sphere with 4 voxels per radius. Note that the number of voxels per object is the critical resolution in the curvature measurement that allows comparison to experiments. The actual physical dimensions of each voxel are arbitrary for these simulations; here, a voxel size of $0.2 \mu \mathrm{m}$ was used to be consistent with the experiment. When single crystal spheres were created within single crystal grains, the smoothing procedure led to a

$\begin{array}{ll}\text { Zhong et al. } & \text { p. } 10\end{array}$ 
significant loss of volume. This corresponds to a shrinking of the radius and an increase in the curvature. The constrained smoothing in DREAM.3D uses nearly stationary triple lines and quadruple points to ameliorate this, so we constructed the spheres at the center of an assembly of eight other grains that all have the same disorientation $\left(45^{\circ} /[100]\right)$ with center grain. So, while there are eight distinct grain boundaries surrounding the sphere, they have crystallographically indistinguishable disorientations.

Two of the spherical grains are illustrated in Fig. 1. An example of a relatively lowresolution sphere (S6) is illustrated in Fig. 1(a). In this case, there are six voxels per radius and the cube shaped voxels make a rather crude approximation of the sphere. A higher resolution sphere (S28) is shown in Fig. 1(d); in this case there are 28 voxels per radius and the spherical shape is much more accurately reproduced. The triangular mesh created by the marching cube algorithm in DREAM.3D has to be smoothed to remove stair-stepped structures. The parameters for the smoothing routine are the number of iterations, $\mathrm{N}$, and a weighting factor, $\lambda$. The weighting factor controls how far a node moves on each iteration and varies between 0 (no smoothing) and 1 (maximum). We tested a number of combinations of $\mathrm{N}$ and $\lambda$, and found that the strength or degree of smoothing increases with the product of these values. Based on these initial tests, and to cover a range of smoothing that seemed practical, we selected four trial values for $\lambda$ and $N$. These conditions have the following labels (values of $\lambda / \mathrm{N}$ ) that correspond to increased smoothing: I $(0.05 / 50)$, II $(0.1 / 100)$, III $(0.2 / 200)$, and IV (0.4/400). The effects of these different parameters are illustrated in Fig. 1. With smoothing I, S6 (Fig. 1(b)) is a fairly good approximation of a sphere. However, voxel-like steps can still be seen on the surface of the higher resolution sphere S28 (Fig. 1(e)), indicating inadequate smoothing. Note that protrusions on the

$\begin{array}{ll}\text { Zhong et al. } & \text { p. } 11\end{array}$ 
spheres show the locations of the triple lines (12) and quad points (6) that were added to reduce shrinkage; smoothing is constrained at these locations. When smoothing III was applied, the higher resolution sphere S28 (Fig. 1(f)) became smooth and sphere-like while the lower resolution sphere, S6 (Fig. 1(c)), was over-smoothed and is more like an octahedron than a sphere. This illustrates that fixed smoothing parameters are not ideal for all grain sizes at a single resolution.

The results for the curvatures computed for the triangles associated with the different spheres and different smoothing processes are summarized in Fig. 2. The dashed line indicates the ideal curvature value calculated from sphere radius $\left(\mathrm{R}^{-1}\right)$ and the markers are the average values of the triangle curvatures for each sphere. The difference between a marker and the corresponding point on the dashed line measures the quality of the reconstruction. The smaller the difference, the better the reconstruction and the better the calculated curvature approximates the true curvature. From the plot, we can see that the optimal smoothing parameters are related to the resolution and feature size. Lower resolution spheres are better smoothed with smaller smoothing parameters while larger smoothing parameters worked better for the higher resolution spheres. This is consistent with the visual interpretation of Fig. 1. The Laplacian smoothing method makes changes in the mesh nodes in proportion to local gradients. The low-resolution sphere (S6) has larger gradients between voxels and was therefore smoothed in a few iterations. The higher resolution sphere, S28, had smaller gradients, so more iterations and larger values of $\lambda$ were required for optimal smoothing. The three exceptions to this trend in Fig. 2 are S4, S6, and S9 with smoothing VI; in this case they were so over smoothed that their shapes collapsed so that they were polyhedral rather than spherical.

$\begin{array}{ll}\text { Zhong et al. } & \text { p. } 12\end{array}$ 

the data and measure the triangle curvatures. It is important to keep in mind that a single resolution value has different impacts on small highly curved and larger flat boundaries within the same microstructure. Also, the effect on specific boundaries is not directly correlated to grain size. To understand the range of curvatures in our data, the distribution of triangle curvatures in the austenitic steel is plotted in Fig. 3 (the distribution for the ferritic steel, not shown, is similar). Though the specific shape of the histogram depends on the smoothing parameters, results given by the four smoothing routines were similar and smoothing II was selected for this example. Because the relevant parameter that affects the curvature measurement is the voxels per grain, the upper horizontal axis in Fig. 3 represents the ideal sphere resolutions, as $\log ($ number of voxels in volume), determined from Fig. 2, that corresponds to the measured curvature in the austenitic steel. The important finding in Fig. 3 is that the number of triangles with large curvatures is small compared to those with small curvatures. In fact, $59.5 \%$ of the triangles have curvatures with absolute values less than $0.5 \mu \mathrm{m}^{-1}$ for the austenitic steel. For ferrite, $55.6 \%$ of the triangle curvatures have absolute values less than $0.5 \mu \mathrm{m}^{-1}$. In other words, most of the grain boundaries in our microstructure maps were relatively flat and were comparable to the surfaces of the larger ideal spheres. Therefore, smoothing II was selected for this study because it gave the best relative accuracy in the majority low curvature boundaries and acceptable accuracy for the higher curvature boundaries. 
When the curvature distribution is considered as a function of any of the crystallographic parameters, it is affected by the discrete binning and symmetry averaging. When the discrete triangles are classified according to their crystallography, they are grouped into bins of finite width and averaged. When the curvature of a particular type of grain boundary is retrieved from these discrete bins, it is the average of 36 separate bins. Therefore, it is reasonable to expect there would be a disparity between triangle curvatures and symmetry averaged curvatures. This effect is not expected to change with the resolution of the mesh.

The curvature distributions at the fixed misorientation between the center sphere and the surrounding grains $\left(45^{\circ} /[100]\right)$ were computed for the ten spheres and the distributions are plotted for S6 and S28 in Fig. 4. For S6 (Fig. 4(a)), the ideal curvature was $0.83 \mu \mathrm{m}^{-1}$ and it should be uniform. The average curvature is somewhat lower $\left(0.65 \mu \mathrm{m}^{-1}\right)$, as expected from Fig. 2, and the individual values vary from $0.22 \mu \mathrm{m}^{-1}$ to $2.8 \mu \mathrm{m}^{-1}$ with the largest outliers arising from the triangles at the triple lines. For the higher resolution case represented by S28, curvature varied in a much smaller range (from $0.11 \mu \mathrm{m}^{-1}$ to $0.26 \mu \mathrm{m}^{-1}$ ) and the average value $\left(0.15 \mu \mathrm{m}^{-1}\right)$ was much closer to its ideal curvature $\left(0.16 \mu \mathrm{m}^{-1}\right)$. The symmetry averaged curvature distribution information for the other spheres are summarized in Fig. 5, which includes the mean triangle curvature (from Fig. 2) and the ideal curvature for comparison. While Fig. 2 illustrates the effects of approximating a curved surface with voxels and allows us to select optimal parameters for the reconstruction, Fig. 5 adds to this the effects of the discrete binning and symmetry averaging on the measured curvature. Based on these tests, we conclude that while high curvatures are underestimated, most curvatures are accurately reproduced. Furthermore, 
when curvature distributions are plotted for real data, the triangles will come from many different grains with different sizes, so that the underestimations of the highest curvature boundaries found on the smallest grains will be ameliorated by averaging with more accurate values from larger grains.

\subsection{The integral mean curvature of grain boundary faces}

To analyze grain boundary curvature as a function of grain size and topology, we consider the curvature integrated over grain faces and entire grains. The integral mean curvatures of grain faces $\left(\mathrm{M}_{\mathrm{s}}\right)$ for every reconstructed grain was calculated using Eq. 3, where $\mathrm{H}_{i j}$ is the mean curvature of the $\mathrm{i}^{\text {th }}$ triangle on the $\mathrm{j}^{\text {th }}$ face of the grain and $\mathrm{S}_{i j}$ is its area. The number of faces of the grain is $\boldsymbol{n}^{f}$ and the number of triangles on one face is $\boldsymbol{n}^{t}$. $\mathrm{M}_{\mathrm{s}}=\sum_{j}^{n^{f}}\left(\sum_{i}^{n^{t}} \mathrm{H}_{i j} \times \mathrm{S}_{i j}\right)$

For the ferrite sample, only the larger of the two volumes containing 1113 grains was analyzed. Furthermore, grains whose centroids fell within $2<R>$ of the free surfaces of the sample, where $\langle R>$ is the average grain radius $(1.18 \mu \mathrm{m}$ for the austenite steel and 1.12 $\mu \mathrm{m}$ for ferrite), were ignored. After this, 1885 grains remained in the austenite and 538 grains remained in the ferrite.

Grains were categorized into topological classes according to their numbers of faces and the results are shown in Fig. 6. The domain of the horizontal axis was limited to 40 to emphasize the points near the zero crossing; this excludes only $3.35 \%$ of the grains. The integral mean curvature was positive for grains with a smaller number of faces and negative for grains with a large number of faces. This is consistent with theories of grain growth that suggest grains with many faces grow and those with few faces shrink. Note

$\begin{array}{ll}\text { Zhong et al. } & \text { p. } 15\end{array}$ 
that for grains within each class, $\mathrm{M}_{\mathrm{s}}$ is a distribution rather than a constant. The ferrite data is noisier than the austenite data, especially for classes with a large number of faces $(>25)$. This is probably because there are fewer grains in the ferrite sample. For ferrite, the classes with F $>25$ faces contain no more than 5 grains. Most of the grains with between 3 and 20 faces and have smaller standard deviations in both datasets. Note that the standard deviation increases again for grains with the fewest faces and the largest curvatures. This is probably because larger curvatures are not measured as accurately as smaller curvatures, as illustrated in Fig. 2. The integral mean curvature crosses zero at about 17 grain faces for both the austenitic and ferritic steel. Changing the minimum grain size did not significantly alter the results.

Rowenhorst et al. [3] proposed that zero curvature grains were those whose numbers of faces (F) were the same as the average numbers of faces of their nearest neighbors, $<\mathrm{F}_{\mathrm{NN}}>$. We tested the same idea on the austenitic steel data (see Fig. 7). The normalized integral mean curvature of grain faces is calculated as $\mathcal{G}^{\prime}=M_{s} / R$, where $R$ is the sphere equivalent radius of the grain. The set of grains for this local topology analysis was limited to ones whose nearest neighbors also fall $2<\mathrm{R}>$ from free surfaces of the sample, leaving 930 grains for analysis in austenite. The grey dots in Fig. 10 are normalized grain curvatures of individual grains. Red squares are average normalized grain curvature for each $\mathrm{F}-<\mathrm{F}_{\mathrm{NN}}>$ class. The line for the average in each class passes almost exactly through the point with $\mathrm{F}-<\mathrm{F}_{\mathrm{NN}}>=0$ and $\mathcal{G}^{\prime}=0$. The results for the ferritic steel are not shown because after grains with incomplete neighbors were also removed, there were not enough grains left to support a statistical analysis. Note that a finite number of grains with two faces were found and observed to be either twins or lens shaped grains at boundaries.

Zhong et al. p. 16 


\subsection{Grain boundary curvature distributions in the austenitic and ferritic steels}

In the remainder of this paper, we refer to the symmetry averaged GBCurD simply as the GBCurD. The simplest projection of the five-parameter GBCurD is the average curvature as a function of disorientation angle, or the smallest misorientation angle among all indistinguishable representations for each triangle. The results for the two samples are shown in Fig. 8, where each point is the average curvature of all of the triangles with that disorientation. The data for the austenite and ferrite have the characteristic that low angle grain boundaries (LAGBs) have curvatures that increase with decreasing disorientation. The exception is for the lowest disorientation angle, which had significantly fewer observations. There is also an increase in curvature at angles near $60^{\circ}$. For the austenite sample, the average curvature of coherent twin boundaries is shown separately and, as expected, this represents a minimum $\left(0.3 \mu \mathrm{m}^{-1}\right)$. The coherent twins make up $14.6 \%$ of the length of all boundaries in the austenite and tend to be very flat and lie on (111) planes. For the ferrite sample, the average curvature of symmetric tilt $(\overline{2} 11) \Sigma 3$ boundaries is also shown. The most populous grain boundary type in BCC ferrite is the ( $\overline{2} 11) \Sigma 3$ boundary; it makes up $\sim 2.5 \%$ of total boundary area. The curvature of the ( $(\overline{2} 11) \Sigma 3$ boundary is not significantly less than the average of other boundaries, even though (as illustrated below) it has the minimum curvature for all $\Sigma 3$ boundaries. When considering these results, keep in mind that at each of the other points, the value is averaged over all disorientation axes and grain boundary plane orientations. Therefore, the higher curvatures at low disorientation angles and near $60^{\circ}$ represent significant trends in the data. Other than these features, the average curvature is approximately constant between $15^{\circ}$ and $55^{\circ}$. The

$\begin{array}{ll}\text { Zhong et al. } & \text { p. } 17\end{array}$ 
fact that the ferrite data set had fewer grains than the austenite data set contributes to the greater scatter in the ferrite curvature distribution.

The data in Fig. 8 suggest that the boundaries at $60^{\circ}$ have curvatures that are slightly greater than at other angles. Also, we know that the singular coherent twin boundary and $\{211\}$ symmetric tilt boundary occur at these disorientations. Furthermore, these samples have more boundaries with this disorientation than any other type, which means that our measurements of these boundaries are the most reliable. Therefore, we examine the curvature as a function of the grain boundary plane orientation for grain boundaries with a $60^{\circ}$ disorientation about the [111] axis $(\Sigma 3)$ for the austenite and ferrite samples. The curvature in Fig. 9 is plotted in stereographic projection with [001] and [100] pointing normal to the plane of the paper and to the right, respectively. The grain boundary populations and relative energies, reported earlier $[14,15]$ are shown for comparison. Note that the grain boundary populations are not identical to those reported earlier because, in this case, they were computed using DREAM.3D [26].

For the austenite sample, the most significant feature appears at the position of the coherent twin. This is the minimum curvature, maximum population, and minimum energy. This is the boundary that is terminated by (111) planes on both sides and is very flat. It is interesting to note that there is a maximum in curvature for boundaries that are $90^{\circ}$ from the twin position and the maximum is reached at the positions of the (110) boundary planes. This is consistent with the observation that twined grains are usually plate-shaped and have large parallel $\{111\}$ faces connected by much smaller perpendicular boundaries. The high curvature results from geometric necessity of joining the two parallel $\{111\}$ planes to bound the grain.

$\begin{array}{ll}\text { Zhong et al. p. } 18 & \end{array}$ 
The distributions for the ferritic sample are nearly opposite that of the austenitic sample. The lowest curvatures occur in the [111] zone with the minimum curvature, energy, and maximum population occurring at the positions of the ( $\overline{2} 11),(\overline{1} \overline{1} 2),(1 \overline{2} 1)$ symmetric tilt grain boundaries (these are indistinguishable grain boundaries). The maximum curvature $\left(1.0 \mu \mathrm{m}^{-1}\right)$ and minimum population occur at the position of the (111) twist grain boundary. It should also be pointed out the range of the area distribution for the austenitic sample is much larger than for the ferritic sample, with a maximum population larger than 300 MRD for the austenitic steel but only about 9 MRD for the ferritic steel.

When comparing the curvature, population, and energy distributions, it seems clear that for both samples, the curvature is correlated to the crystallography of the grain boundary, as hypothesized in $\S 1$. Furthermore, the comparison of curvature and energy shows systematic variations. The two boundaries thought to be singular, the (111) $\Sigma 3$ boundary in austenite and the ( $\overline{2} 11) \Sigma 3$ boundary in ferrite, have the minimum curvature, minimum energy, and maximum population. This implies a direct correlation between curvature, energy, and population. However, this is not the case at all points. For example, if we compare the energy and curvature of the grain boundaries in the [111] zone for austentite (see Fig. 10) we see an inverse correlation between energy and curvature. Because these are among the highest energy and lowest population of the $\Sigma 3$ grain boundaries, we also assume that these are non-singular orientations.

Zhong et al. 


\section{Discussion}

The results in $\S 3.1$ indicate that measuring curvatures from a discrete triangular mesh using the method of Goldfeather and Interrante [31] is accurate provided that there is sufficient resolution compared to the range of curvature to be measured. A basic limitation will always be that there is a wide range of grain sizes with different curvatures and no single smoothing procedure will be ideal for all grains. Fortunately, the largest fraction of boundaries has relatively low curvatures that are more accurately measured and these will dominate the distribution. For example, the curvatures of specific grain boundary types, as illustrated in Figs. 8 through 10, will be the average of values from the majority low curvature grain boundaries that are accurately measured and the minority high curvature boundaries whose curvatures are underestimated. So, the while the underestimated curvature of some boundaries will affect the results, the effect will be diluted by the majority boundaries that are measured more accurately.

Because the curvature-energy product is the driving force for grain growth, there is an extensive literature, dating back to the work of Smith, [34] relating grain topology, integral mean curvature, and the tendency of grains to grow or shrink. Theoretical treatments of the problem led to the prediction that grains with more than 13 or 14 faces grow and those with fewer shrink. [35-37] Rowenhorst et al. [3] reported that zero curvature grains had 15.5 faces, Mason et al. [38], based on simulations of isotropic grain growth, found the crossover from negative to positive to take place around 15 faces, and in the current work we find it is about 17. The difference between the value we report and that reported by Rowenhorst et al. [3] might not be significant. Note that while the data follow the trend of decreasing integral mean curvature with an increasing number of faces, the curvatures of

$\begin{array}{ll}\text { Zhong et al. } & \text { p. } 20\end{array}$ 
grains with between 15 and 20 faces are less than the standard deviation, so the exact point where the curvature is zero is not clear. We note that the average numbers of faces per grain in the austenite and ferrite was 13.6 and 13.7, respectively, which are consistent with the number reported for $\beta$-Ti (13.7) [3] and similar to that reported for $\alpha$-Ti (14.2). [39]

MacPherson and Srolovitz $[40,41]$ recently showed that the number of faces alone does not determine the growth rate of a grain. Using data from more than $2000 \beta$-Ti grains, Rowenhorst et al. [3] demonstrated the validity of the idea that zero curvature grains were those whose numbers of faces (F) were the same as the average numbers of faces of their nearest neighbors, $\left\langle\mathrm{F}_{\mathrm{NN}}>\right.$. The fact that we get the same result from an austenitic steel (see Fig. 7), which has a significantly different microstructure, suggests that this is a general feature of microstructures undergoing grain growth.

The observations reported in Figs. 8 to 10 provide clear evidence that the mean curvature of the grain boundary depends on its crystallographic parameters. In an isotropic system, one would expect the curvature be dictated by the size of the grain face. While it seems sensible that the grain and face size will still play a role, there is a clear effect of both lattice disorientation (Fig. 8) and the grain boundary plane orientation (Fig. 9 and 10). In Fig. 9(a), the curvature for the $60^{\circ} /[111]$ boundary in austenite varies from 0.3 $\mu \mathrm{m}^{-1}$ at the position of the (111) twist boundary to $1.2 \mu \mathrm{m}^{-1}$ at the (110)-type positions $90^{\circ}$ away. There are also significant curvature variations for the $60^{\circ} /[111]$ boundary in ferrite, even though the anisotropy in the grain boundary population is 30 times smaller. The variations with grain boundary plane at both of these disorientations are greater than the average values at different disorientations (see Fig. 8).

$\begin{array}{ll}\text { Zhong et al. } & \text { p. } 21\end{array}$ 
The data in Fig. 6 show that there is a strong correlation between curvature and the number of faces. Because it is known that grains with more faces are larger, there is also an intuitive correlation between curvature and grain size. $[42,3,39]$ When the GBCurD is computed, all grains are included. This means that for a single crystallographic type, triangles from small, higher curvature, shrinking grains are averaged together with triangles from large, lower curvature, growing grains. Despite this, there is still a crystallographic correlation. One might think this is because the GBCurD is area weighted, so the larger grains have a more significant effect on the distribution than small grains. However, the large growing grains necessarily share boundaries with smaller shrinking grains, so one cannot attribute the distribution solely to the large grains.

While the GBCurD is clearly sensitive to crystallography, there is no single correlation between curvature and energy or curvature and population. The one trend that is clear is that the lowest energy boundaries also have small curvature and large areas. If these boundaries are presumed to be singular and in contact the same mean field chemical potential, then the curvature should be inversely proportional to the energies of the boundaries peripheral to the singular surface. Such boundaries are always have higher energies than the singular surface, leading to a director proportionality between the energy of the singular surface and the curvature. This is certainly obvious from the distributions plotted in Fig. 9. However, there are also examples of opposite correlations. One example is clearly illustrated in Fig. 8, which shows that low disorientation angle grain boundaries have curvatures that increase as the disorientation decreases. It is well known that the grain boundary energy decreases at low disorientation angles, [43] where the curvature is observed to increase. A second example is illustrated in Fig. 10. As noted in $§ 1$, non-

$\begin{array}{ll}\text { Zhong et al. } & \text { p. } 22\end{array}$ 
singular boundaries in contact with a constant mean field chemical potential should have curvatures that are inversely correlated to the energy.

Some of the preceding arguments are based on a mean field chemical potential for the grain boundaries. It should be noted that each individual boundary is not assumed to have the same chemical potential. However, computing the curvature of a certain type of boundary involves averaging over many boundaries at different chemical potentials. The average curvature that results is therefore representative of mean chemical potential of the boundaries with the same crystallography.

The data in Fig. 9 and 10 show only a small portion of all grain boundary types. When the data is examined for all grain boundaries, the curvature is not strongly correlated to the population or energy, although the curvature and energy have a weak negative correlation. The absence of a strong correlation may be because of the competing behaviors of the singular and non-singular interfaces. Unfortunately, at the present time, is it not obvious how to classify the boundary types into these categories. It should also be pointed out the lower the population of a boundary, the greater the uncertainty in the curvature and energy measurements, so this may also play a role and mask any correlations that might exist. What we can say with confidence is that for the most commonly observed grain boundaries, the dominant trend is that low curvature boundaries have low energy and larger areas. However, there are no strong overall correlations between curvature and energy or area, similar to the area-energy correlation that was observed in many materials. [16]

Zhong et al. p. 23 


\section{Conclusions}

Grain boundary mean curvatures can be accurately measured from discrete triangular meshes, but the highest curvatures are underestimated. The curvatures measured for an austenitic steel and a ferritic steel show the expected trend that the integral mean curvature of the grains decreases as the number of grain faces increases. The curvatures are also related to grain topology. In austenite, when the number of faces on a grain is equal to the average number of faces of its neighbors, it has zero integral mean curvature. We find that the crystallography of the grain boundary strongly influences the curvature. The variation of the curvature with the grain boundary plane orientation is stronger than the variation with grain boundary disorientation angle. The lowest curvature grain boundaries also have the lowest grain boundary energies and highest grain boundary areas. However, when all grain boundaries are considered, the curvature is not strongly correlated to energy or area and this might be the result of conflicting mechanisms that determine the curvatures of singular and non-singular boundaries.

\section{Acknowledgements}

G.S.R. acknowledges support from the National Science Foundation under MRI grant DMR 1428480. D.J.R. Acknowledges the support from the Office Naval Research under the Structural Materials Program. The authors acknowledge use of the Materials Characterization Facility at Carnegie Mellon University supported by grant MCF-677785. A part of this work was conducted with the support of the Deakin Advanced Characterization Facility. 


\section{Figure Captions}

Figure 1. Shapes of spherical grains S6 and S28 after different reconstruction procedures. Initial structure of S6 (a) and S28 (d). Shape of S6 (b) and S28 (e) after smoothing I. Shape of S6 (c) and S28 (f) after smoothing III.

Figure 2. Average triangle curvatures for spheres of different resolutions and smoothing parameters. The bars represent the standard deviations of triangle curvatures when spheres are smoothed with smoothing II.

Figure 3. Triangle curvature frequency in the austenitic steel, produced with smoothing II.

Figure 4. (a) Curvature distribution of S6, (b) Curvature distribution of S28.

Figure 5. Average curvature of the distribution for the 10 spheres when smoothing II is used for the reconstruction. The dots are mean symmetry averaged curvatures. The squares are mean triangle curvature. The error bars are standard deviations.

Figure 6. Integral mean curvature of grain faces $\left(M_{s}\right)$ as a function of number of faces of grains (F). The markers represent average $\mathrm{M}_{\mathrm{s}}$ for each grain class and the error bars show the standard deviation within the grain class.

Figure 7. Normalized integral curvature of grain faces $\left(\mathcal{G}^{\prime}\right)$ as a function of $\mathrm{F}-<\mathrm{F}_{\mathrm{NN}}>$ in austenite. $\mathrm{F}-<\mathrm{F}_{\mathrm{NN}}>$ is difference between number of faces of one grain $(\mathrm{F})$ and the average number of faces of its nearest neighbors $\left(<\mathrm{F}_{\mathrm{NN}}>\right)$.

Figure 8. Average curvature as a function of disorientation angle in (a) austenitic steel, (b) ferritic steel.

Figure 9. Curvature (a, d), population (b, e), and energy (c, f) distributions for austenite (ac) and ferrite (d-f) for all grain boundaries with a $\Sigma 3\left(60^{\circ} /[111]\right)$ disorientation. The small

Zhong et al. $\quad$ p. 25 
squares are for reference, marking $\{110\}$ locations in the [111] zone in (a-c) and are

omitted from (d-f) for clarity.

Figure 10. Comparison of the curvature and relative energies of grain boundaries in the

[111] zone of austenite, beginning at the (110) orientation. Because of bicrystal symmetry, the values repeat in periods of $60^{\circ}$.

\section{References Cited}

[1] M.A. Groeber, B.K. Haley, M.D. Uchic, D.M. Dimiduk, S. Ghosh. 3D reconstruction and characterization of polycrystalline microstructures using a FIB-SEM system, Mater. Charact. 57 (2006) 259-273. http://dx.doi.org/10.1016/j.matchar.2006.01.019

[2] M.D. Uchic, M.A. Groeber, D.M. Dimiduk, J.P. Simmons. 3D microstructural characterization of nickel superalloys via serial-sectioning using a dual beam FIB-SEM, Scripta Mater. 55 (2006) 23-28. http://dx.doi.org/10.1016/j.scriptamat.2006.02.039 [3] D.J. Rowenhorst, A.C. Lewis, G. Spanos. Three-dimensional analysis of grain topology and interface curvature in a beta-titanium alloy, Acta Mater. 58 (2010) 5511-5519. http://dx.doi.org/10.1016/j.actamat.2010.06.030

[4] D.M. Saylor, A. Morawiec, G.S. Rohrer. Distribution and energies of grain boundaries in magnesia as a function of five degrees of freedom, J. Am. Ceram. Soc. 85 (2002) 30813083.

[5] B. Lin, Y. Jin, C.M. Hefferan, S.F. Li, J. Lind, R.M. Suter, M. Bernacki, N. Bozzolo, A.D. Rollett, G.S. Rohrer. Observation of annealing twin nucleation at triple lines in nickel during grain growth, Acta Mater. 99 (2015) 63-68.

http://dx.doi.org/10.1016/j.actamat.2015.07.041

[6] D.M. Saylor, A. Morawiec, G.S. Rohrer. The relative free energies of grain boundaries in magnesia as a function of five macroscopic parameters, Acta Mater. 51 (2003) 36753686. http://dx.doi.org/10.1016/S1359-6454(03)00182-4

[7] G. Rohrer, D. Saylor, B. El Dasher, B. Adams, A. Rollett, P. Wynblatt. The distribution of internal interfaces in polycrystals, Zeitschrift Fur Metallkunde 95 (2004) 197-214.

[8] V. Randle. Twinning-related grain boundary engineering, Acta Mater. 52 (2004) 4067-4081. http://dx.doi.org/10.1016/j.actamat.2004.05.031

[9] C.S. Kim, A.D. Rollett, G.S. Rohrer. Grain boundary planes: New dimensions in the grain boundary character distribution, Scripta Mater. 54 (2006) 1005-1009. http://dx.doi.org/10.1016/j.scriptamat.2005.11.071

[10] V. Randle. Role of grain boundary plane in grain boundary engineering, Mater. Sci. Tech. 26 (2010) 774-780. http://dx.doi.org/10.1179/026708309x12567268926641 [11] S.J. Dillon, G.S. Rohrer. Characterization of the Grain-Boundary Character and Energy Distributions of Yttria Using Automated Serial Sectioning and EBSD in the FIB, J. Am. Ceram. Soc. 92 (2009) 1580-1585. http://dx.doi.org/10.1111/j.1551-2916.2009.03064.x

$\begin{array}{ll}\text { Zhong et al. } & \text { p. } 26\end{array}$ 
[12] J. Li, S.J. Dillon, G.S. Rohrer. Relative grain boundary area and energy distributions in nickel, Acta Mater. 57 (2009) 4304-4311.

http://dx.doi.org/10.1016/j.actamat.2009.06.004

[13] G.S. Rohrer, J. Li, S. Lee, A.D. Rollett, M. Groeber, M.D. Uchic. Deriving grain boundary character distributions and relative grain boundary energies from three-dimensional EBSD data, Mater. Sci. Tech. 26 (2010) 661-669. http://dx.doi.org/10.1179/026708309x12468927349370

[14] H. Beladi, G.S. Rohrer. The relative grain boundary area and energy distributions in a ferritic steel determined from three-dimensional electron backscatter diffraction maps, Acta Mater. 61 (2013) 1404-1412. http://dx.doi.org/10.1016/j.actamat.2012.11.017 [15] H. Beladi, N.T. Nuhfer, G.S. Rohrer. The five-parameter grain boundary character and energy distributions of a fully austenitic high-manganese steel using three dimensional data, Acta Mater. 70 (2014) 281-289. http://dx.doi.org/10.1016/j.actamat.2014.02.038 [16] G.S. Rohrer. Grain boundary energy anisotropy: a review, Journal of Materials Science 46 (2011) 5881-5895. http://dx.doi.org/10.1007/s10853-011-5677-3

[17] M. Hillert. On theory of normal and abnormal grain growth, Acta Metall. 13 (1965) 227-238. http://dx.doi.org/10.1016/0001-6160(65)90200-2

[18] J. Jeppsson, J. Agren, M. Hillert. Modified mean field models of normal grain growth, Acta Mater. 56 (2008) 5188-5201. http://dx.doi.org/10.1016/j.actamat.2008.06.034

[19] C. Herring. Surface Tension as a Motivation for Sintering. in: Kingston WE, (Ed.). The Physics of Powder Metallurgy. McGraw-Hill, New Your, 1951. pp. 36.

[20] C. Herring. The Use of Classical Macroscopic Concepts in Surface Energy Problems. in: Gomer R, Smith CS, (Eds.). Structure and Properties of Solid Surfaces; proceedings of a conference arranged by the National Research Council and held in September, 1952, in Lake Geneva, Wisconsin, USA. University of Chicago Press, Chicago, 1953. pp. 5-81.

[21] W.W. Mullins. Capillarity-induced surface morphologies, Int. Sci. 9 (2001) 9-20. http://dx.doi.org/10.1023/a:1011258510496

[22] G.S. Rohrer, E.A. Holm, A.D. Rollett, S.M. Foiles, J. Li, D.L. Olmsted. Comparing calculated and measured grain boundary energies in nickel, Acta Mater. 58 (2010) 50635069. http://dx.doi.org/10.1016/j.actamat.2010.05.042

[23] C. Herring. SOME THEOREMS ON THE FREE ENERGIES OF CRYSTAL SURFACES, Physical Review 82 (1951) 87-93. http://dx.doi.org/10.1103/PhysRev.82.87

[24] J.E. Taylor. Mean-curvature and weighted mean-curvature. 2, Acta Metall. Mater. 40 (1992) 1475-1485. http://dx.doi.org/10.1016/0956-7151(92)90091-r

[25] G.S. Rohrer. Grain Boundary Data Archive.

http://mimp.materials.cmu.edu/ gr20/Grain_Boundary_Data_Archive/, 2015.

[26] M.A. Groeber, M.A. Jackson. DREAM.3D: A Digital Representation Environment for the Analysis of, Microstructure in 3D, 2014, 3:5, Integrating Materials and Manufacturing Innovation 3 (2014) 5.

[27] S.I. Wright, M.M. Nowell. EBSD image quality mapping, Microscopy and Microanalysis 12 (2006) 72-84. http://dx.doi.org/10.1017/s1431927606060090

[28] Y. Bhandari, S. Sarkar, M. Groeber, M.D. Uchic, D.M. Dimiduk, S. Ghosh. 3D polycrystalline microstructure reconstruction from FIB generated serial sections for FE analysis, Computational Materials Science 41 (2007) 222-235. http://dx.doi.org/10.1016/j.commatsci.2007.04.007 
[29] S. Ghosh, Y. Bllandari, M. Groeber. CAD-based reconstruction of 3D polycrystalline alloy microstructures from FIB generated serial sections, Computer-Aided Design 40 (2008) 293-310. http://dx.doi.org/10.1016/j.cad.2007.11.003

[30] B.R. Patterson, D.J. Rowenhorst, V. Tikare, R.T. DeHoff, T.M. Kaub. Affinities for topological arrangements in grain structures, Acta Materialia 79 (2014) 411-420. http://dx.doi.org/10.1016/j.actamat.2013.10.020

[31] J. Goldfeather, V. Interrante. A novel cubic-order algorithm for approximating principal direction vectors, ACM Trans. Graph. 23 (2004) 45-63. http://dx.doi.org/10.1145/966131.966134

[32] A. Morawiec. Method to calculate the grain boundary energy distribution over the space of macroscopic boundary parameters from the geometry of triple junctions, Acta Mater. 48 (2000) 3525-3532.

[33] D.M. Saylor, A. Morawiec, G.S. Rohrer. Distribution of grain boundaries in magnesia as a function of five macroscopic parameters, Acta Mater. 51 (2003) 3663-3674.

http://dx.doi.org/10.1016/s1359-6454(03)00181-2

[34] C.S. Smith. Grains, phases, and interfaces - an interpretation of microstructure, T. Am. I. Min. Met. Eng. 175 (1948) 15-51.

[35] W.W. Mullins. Estimation of the geometrical rate-constant in idealized 3 dimensional grain-growth, Acta Metall. 37 (1989) 2979-2984. http://dx.doi.org/10.1016/0001-6160(89)90333-7

[36] S. Hilgenfeldt, A.M. Kraynik, S.A. Koehler, H.A. Stone. An accurate von Neumann's law for three-dimensional foams, Phys. Rev. Lett. 86 (2001) 2685-2688.

http://dx.doi.org/10.1103/PhysRevLett.86.2685

[37] M.E. Glicksman. Analysis of 3-D network structures, Phil. Mag. 85 (2005) 3-31. http://dx.doi.org/10.1080/14786430412331329892

[38] J.K. Mason, E.A. Lazar, R.D. MacPherson, D.J. Srolovitz. Geometric and topological properties of the canonical grain-growth microstructure, Physical Review E 92 (2015). http://dx.doi.org/10.1103/PhysRevE.92.063308

[39] M.N. Kelly, K. Glowinski, N.T. Nuhfer, G.S. Rohrer. The five parameter grain boundary character distribution of alpha-Ti determined from three-dimensional orientation data, Acta Mater. 111 (2016) 22-30. http://dx.doi.org/10.1016/j.actamat.2016.03.029 [40] R.D. MacPherson, D.J. Srolovitz. The von Neumann relation generalized to coarsening of three-dimensional microstructures, Nature 446 (2007) 1053-1055. http://dx.doi.org/10.1038/nature05745 [41] E.A. Lazar, J.K. Mason, R.D. MacPherson, D.J. Srolovitz. A more accurate threedimensional grain growth algorithm, Acta Mater. 59 (2011) 6837-6847. http://dx.doi.org/10.1016/j.actamat.2011.07.052

[42] F.C. Hull. Plane section and spatial characteristics of equiaxed beta-brass grains, Mater. Sci. Tech. 4 (1988) 778-785.

[43] W.T. Read, W. Shockley. Dislocation models of crystal grain boundaries, Phys. Rev. 78 (1950) 275-289. 

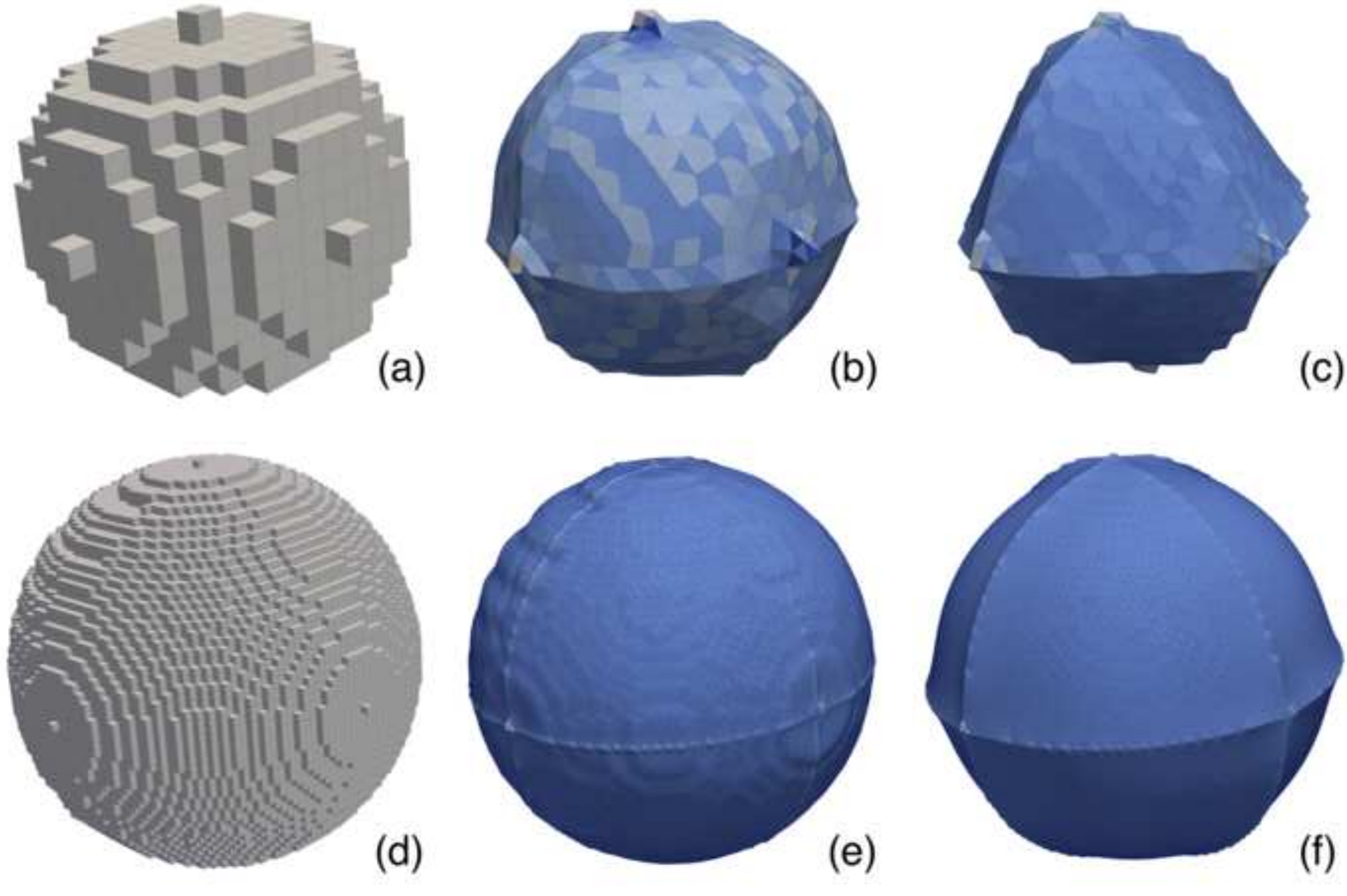

(a)

(b)

(c)

(d)

(e)

(f) 
Sphere Radius (voxels)

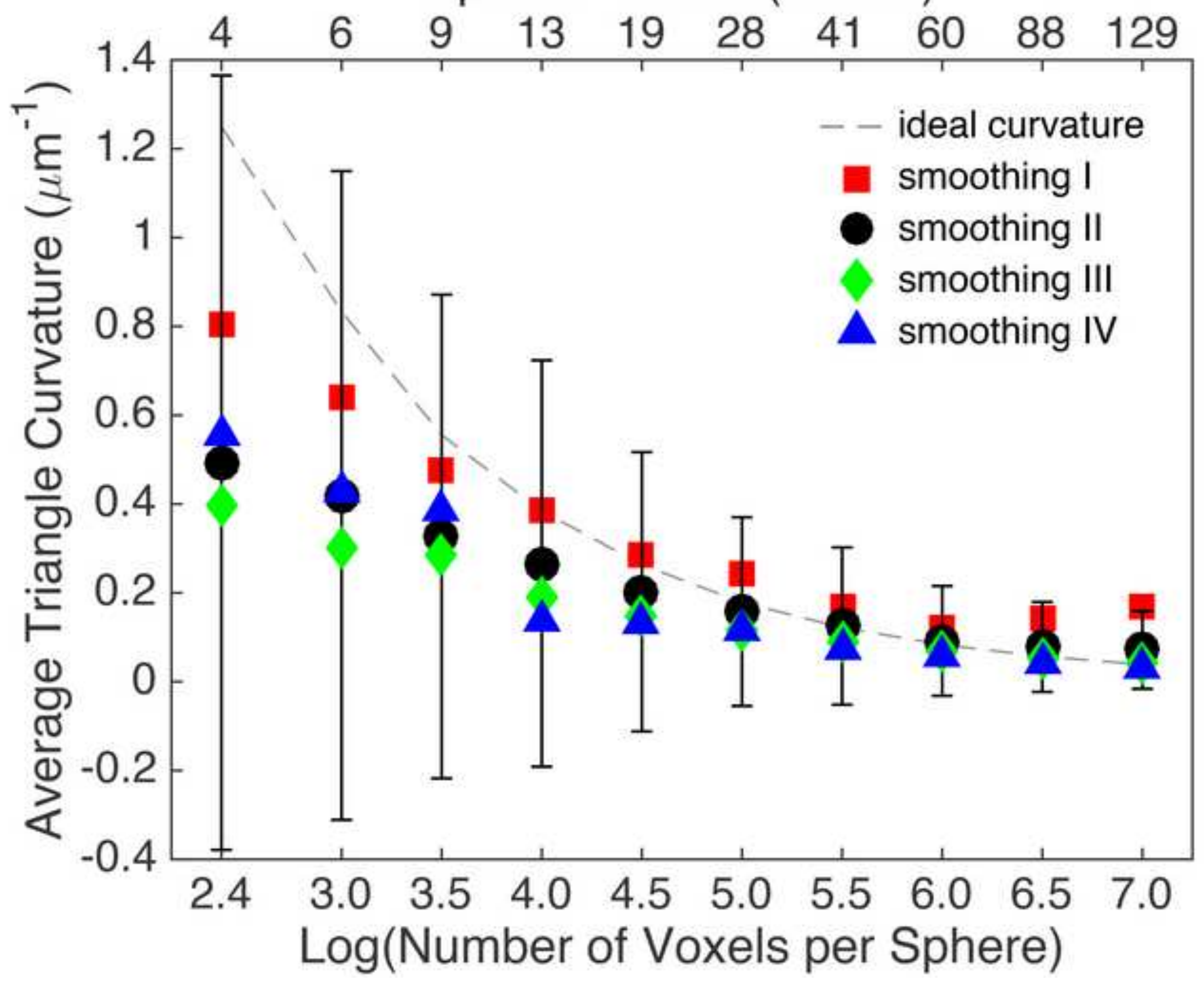




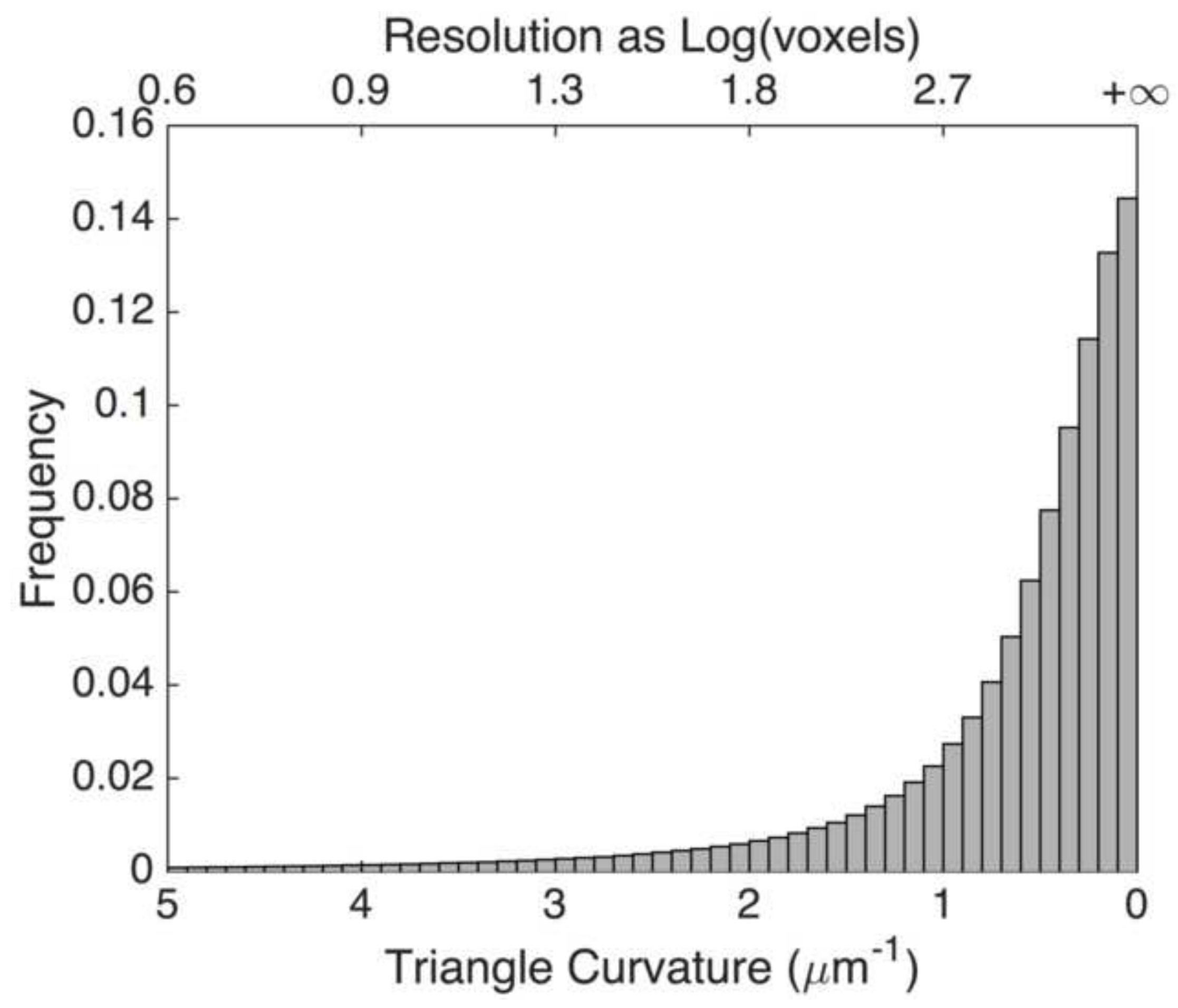

Resolution as Log(voxels) 

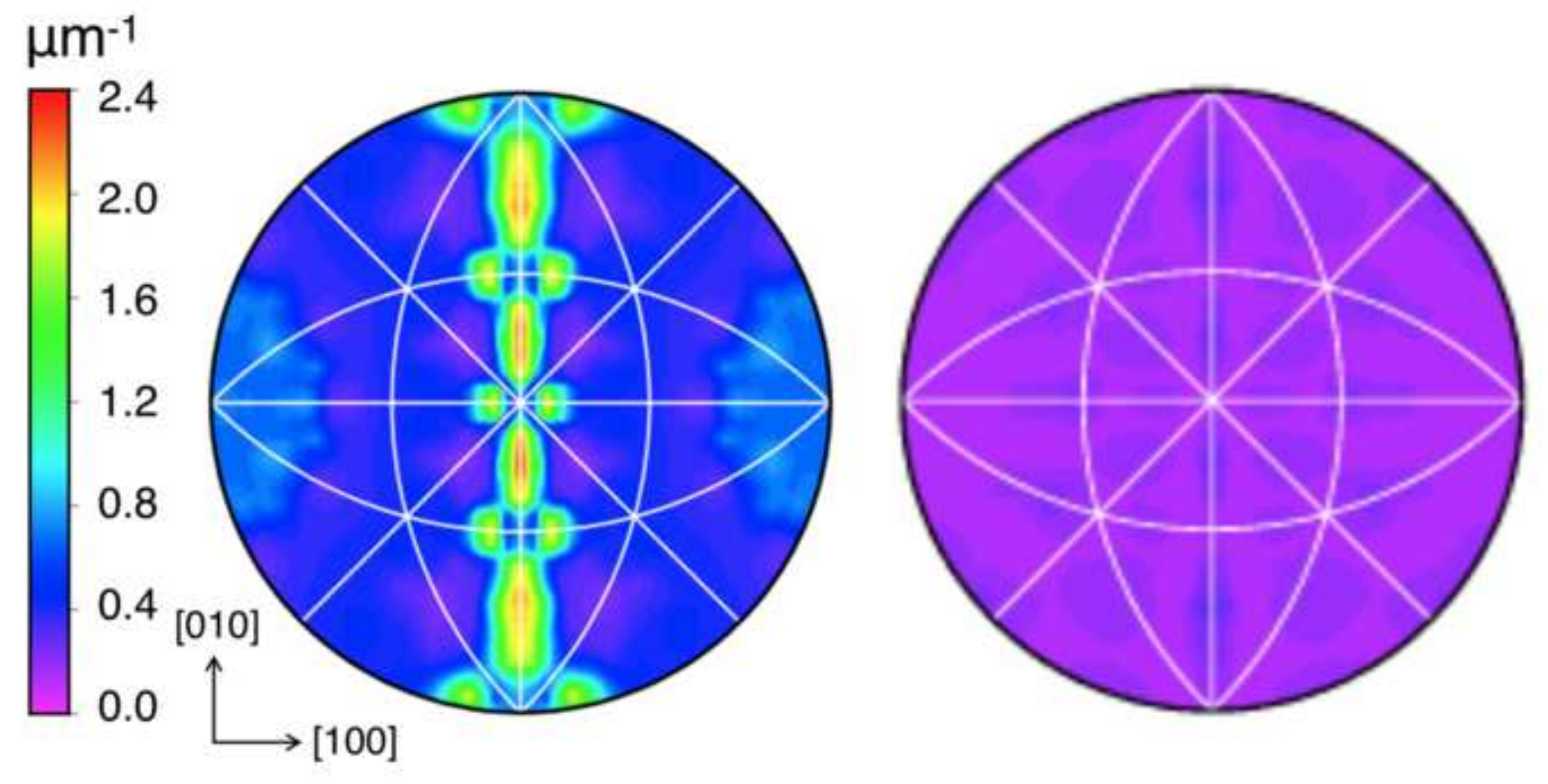


\section{Sphere Radius (voxels)}

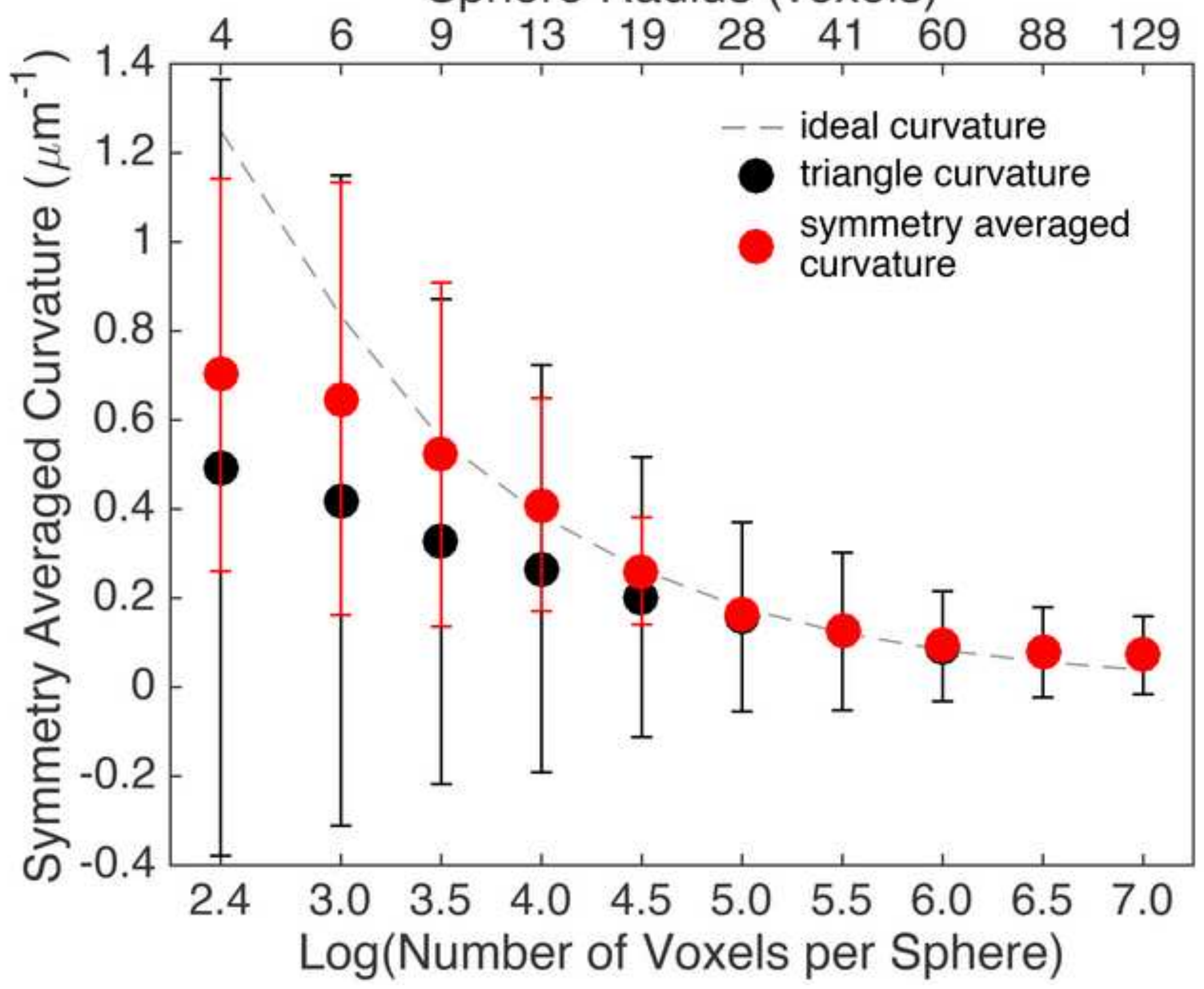




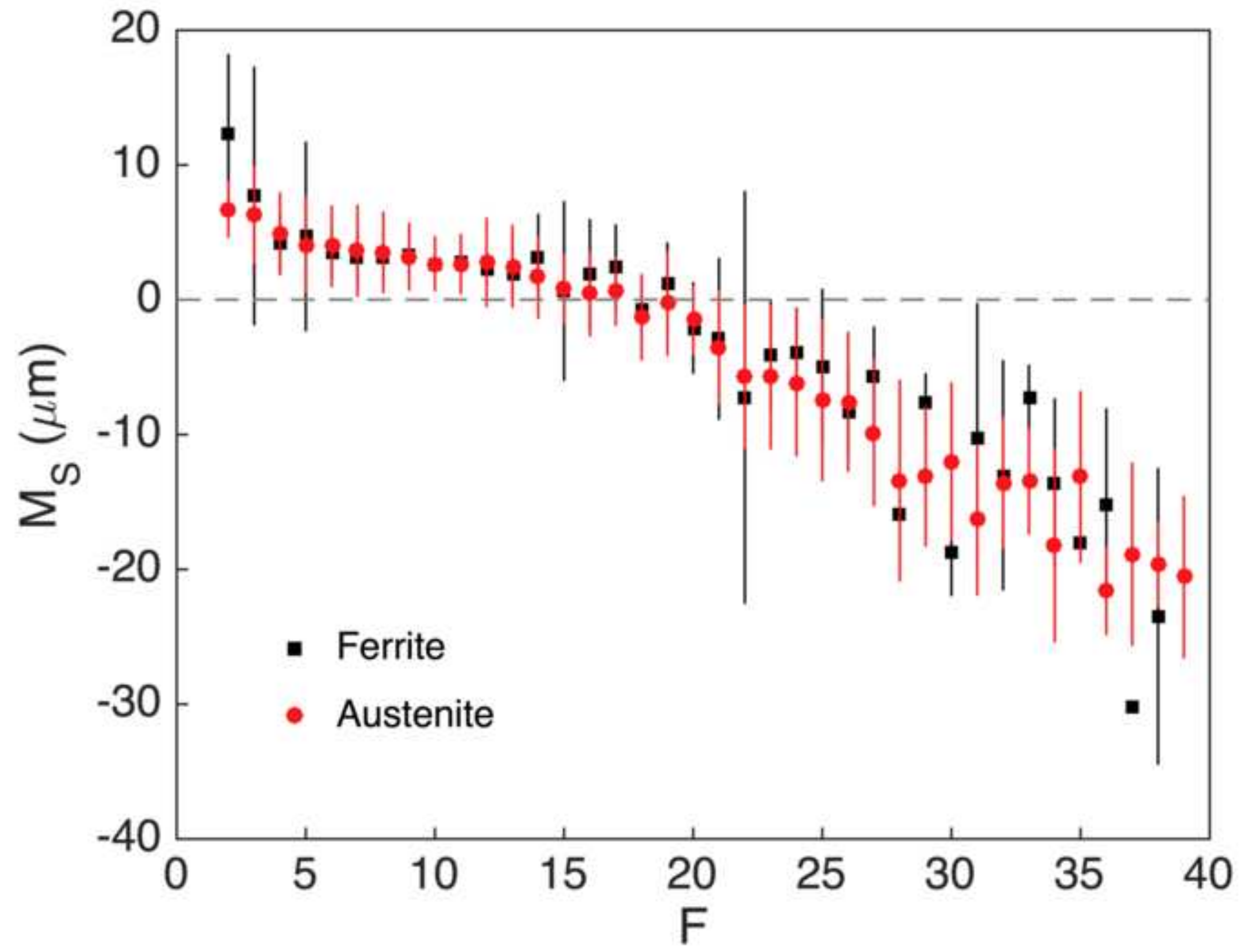




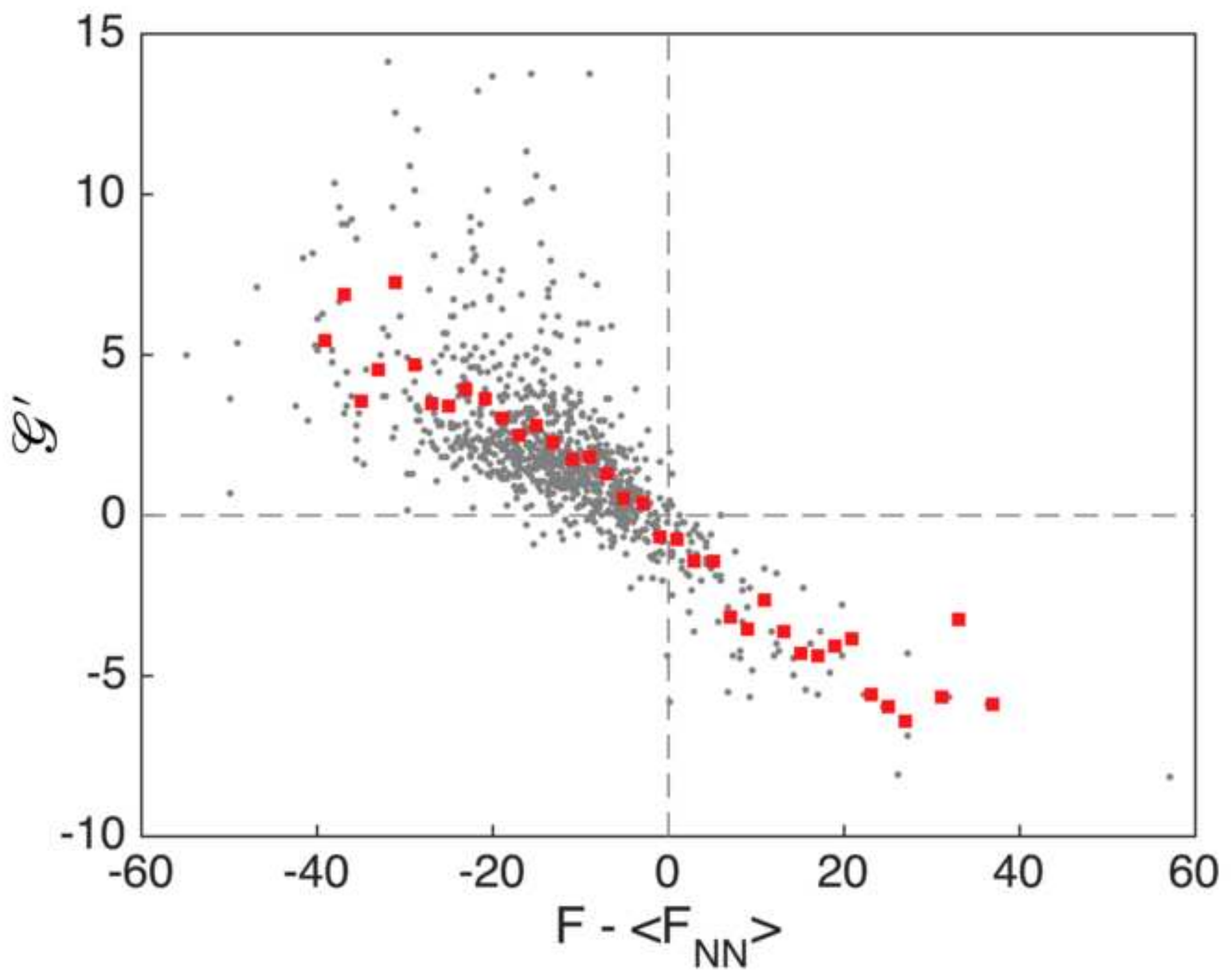



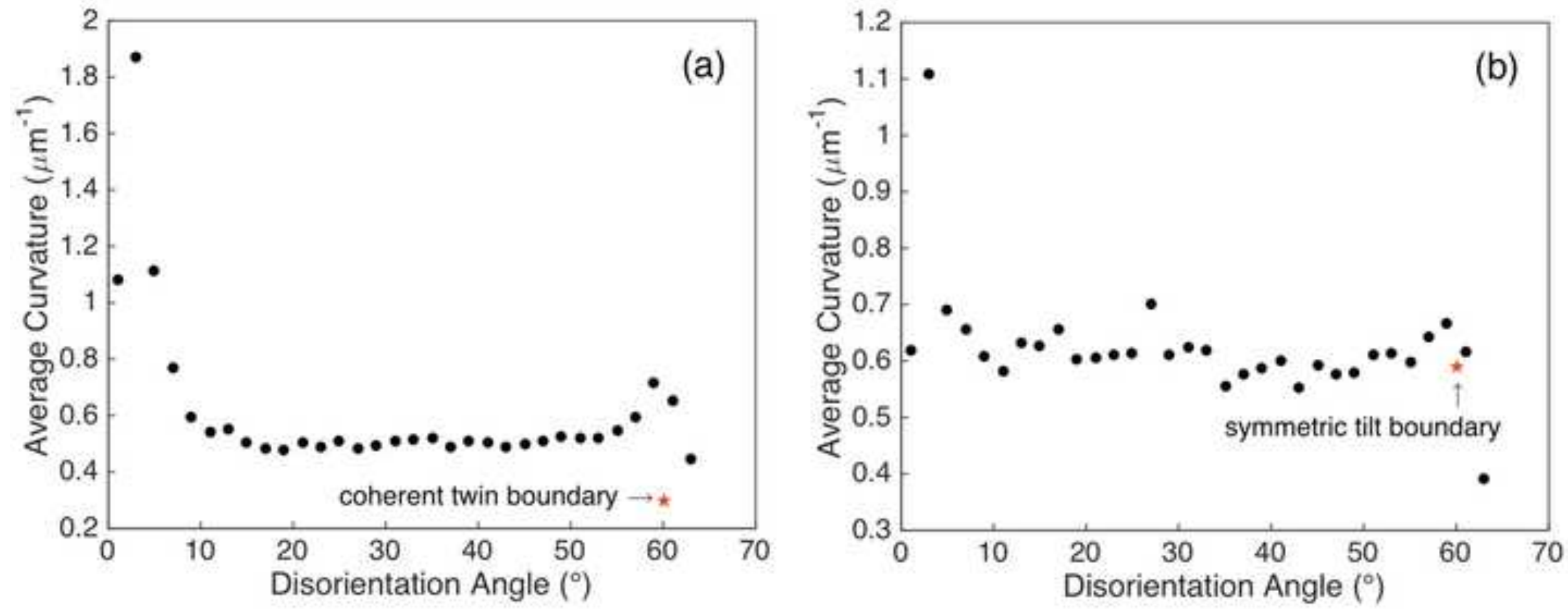

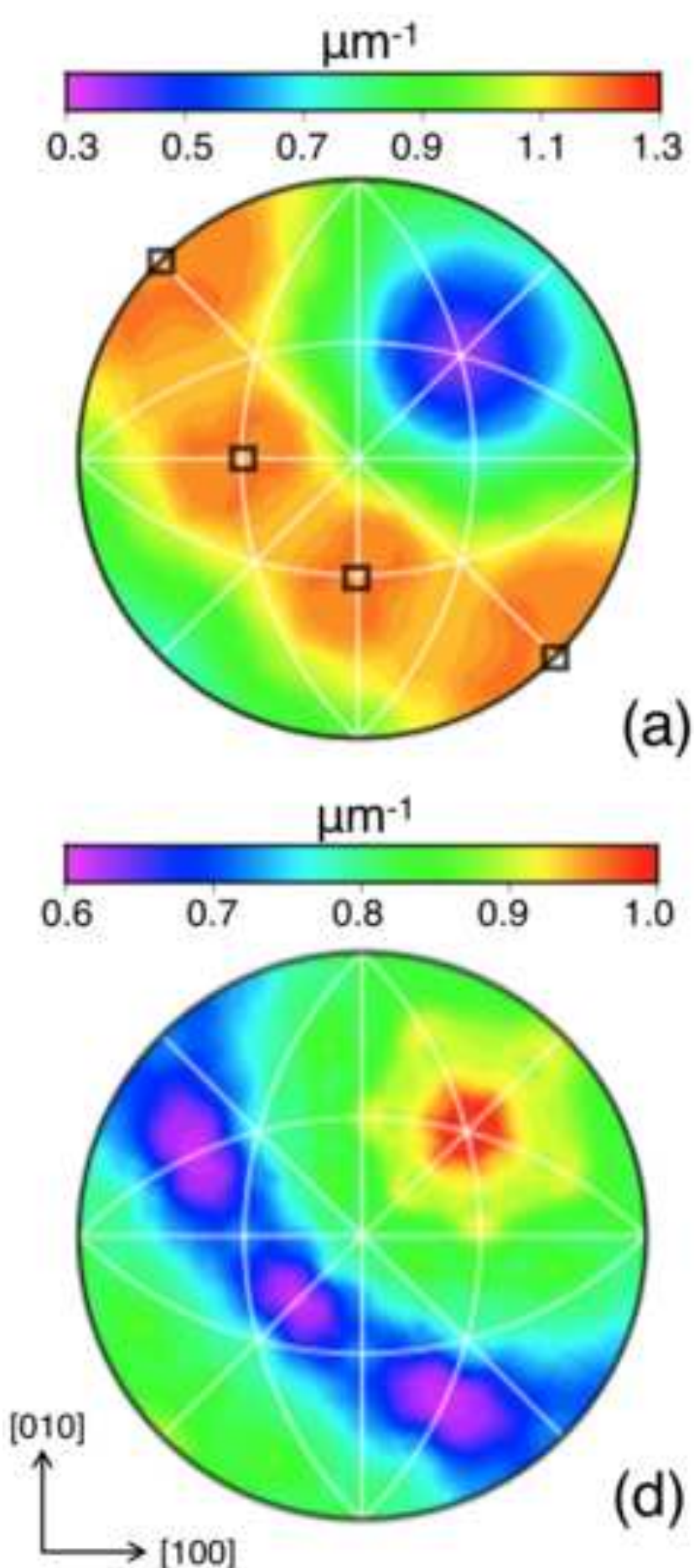

a)
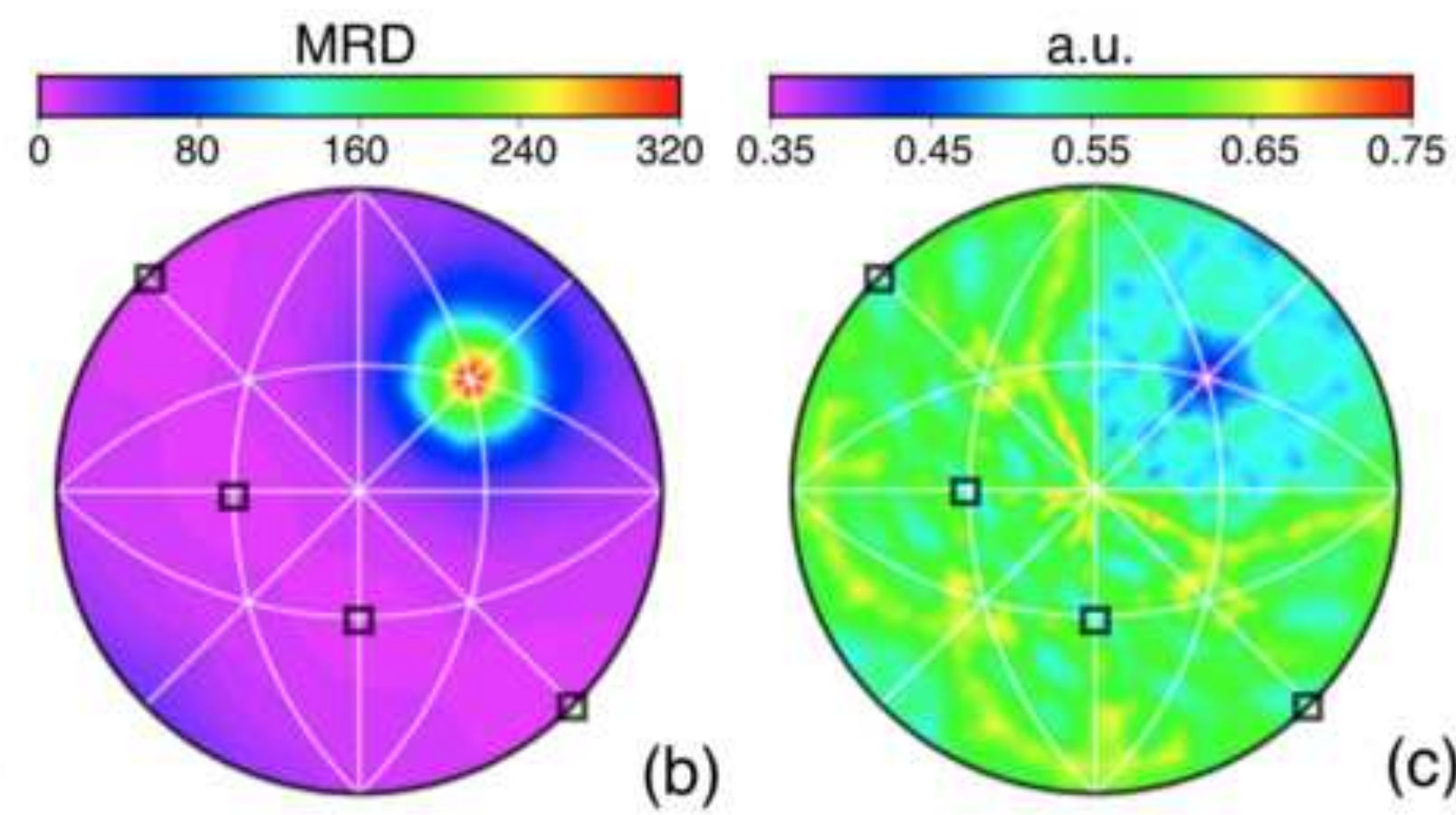

(b)

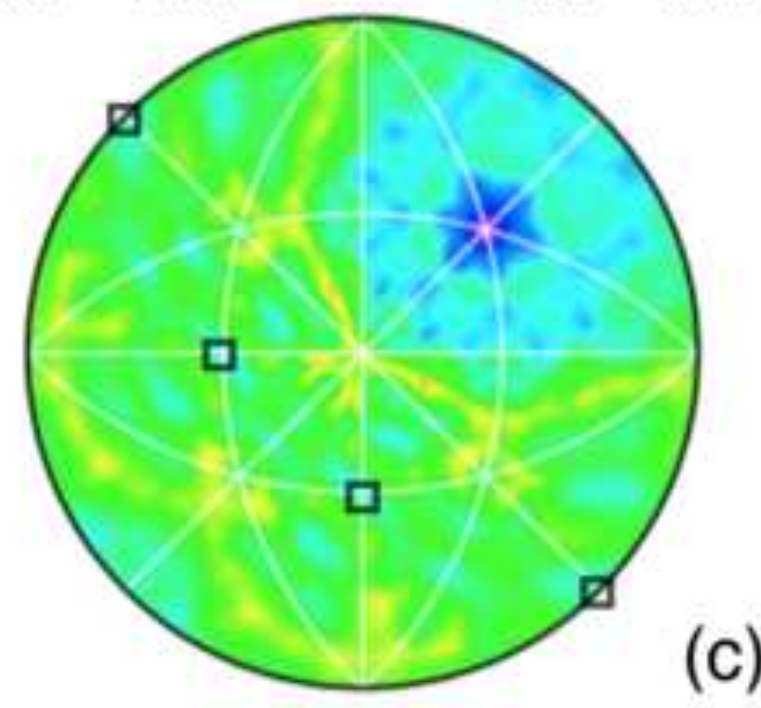

MRD
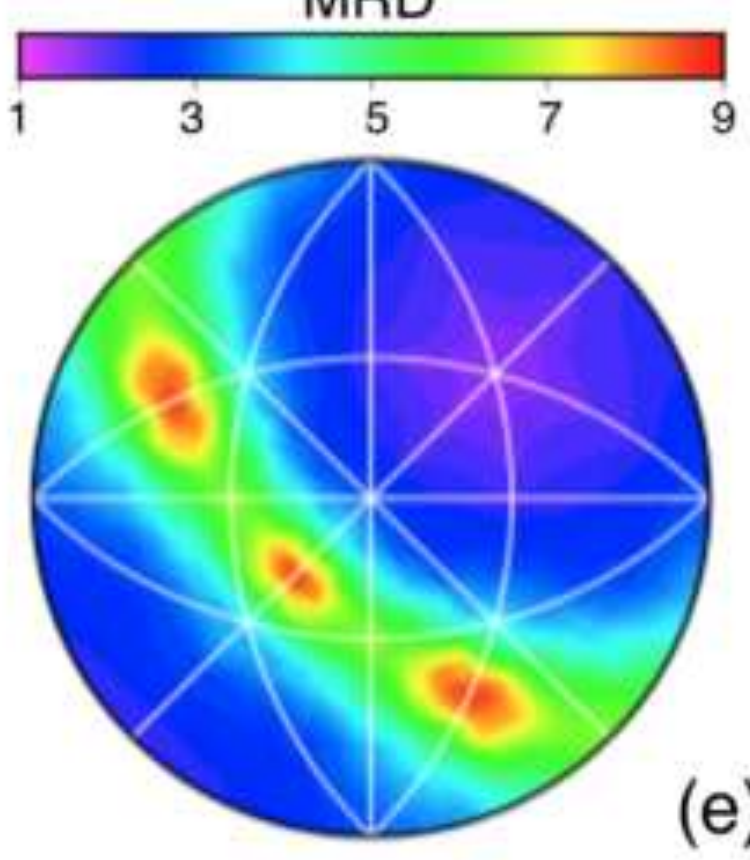

(e)
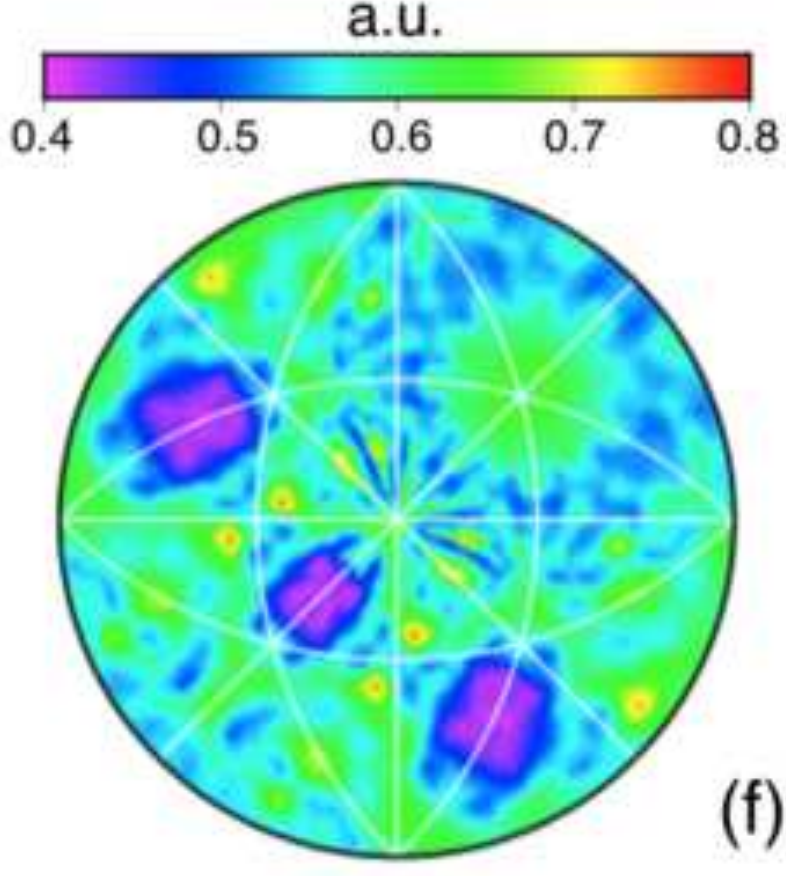
Grain Boundary Planes

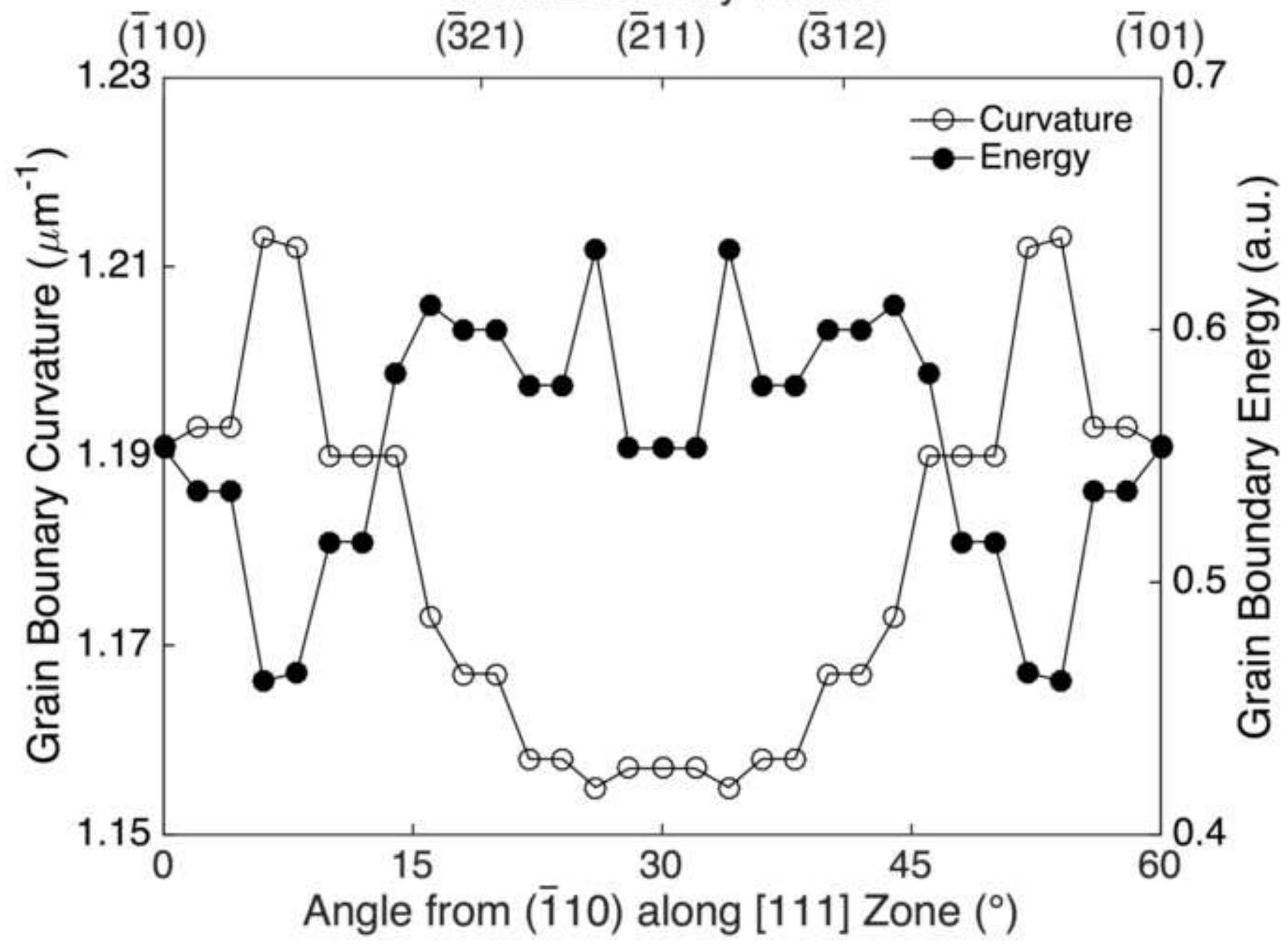




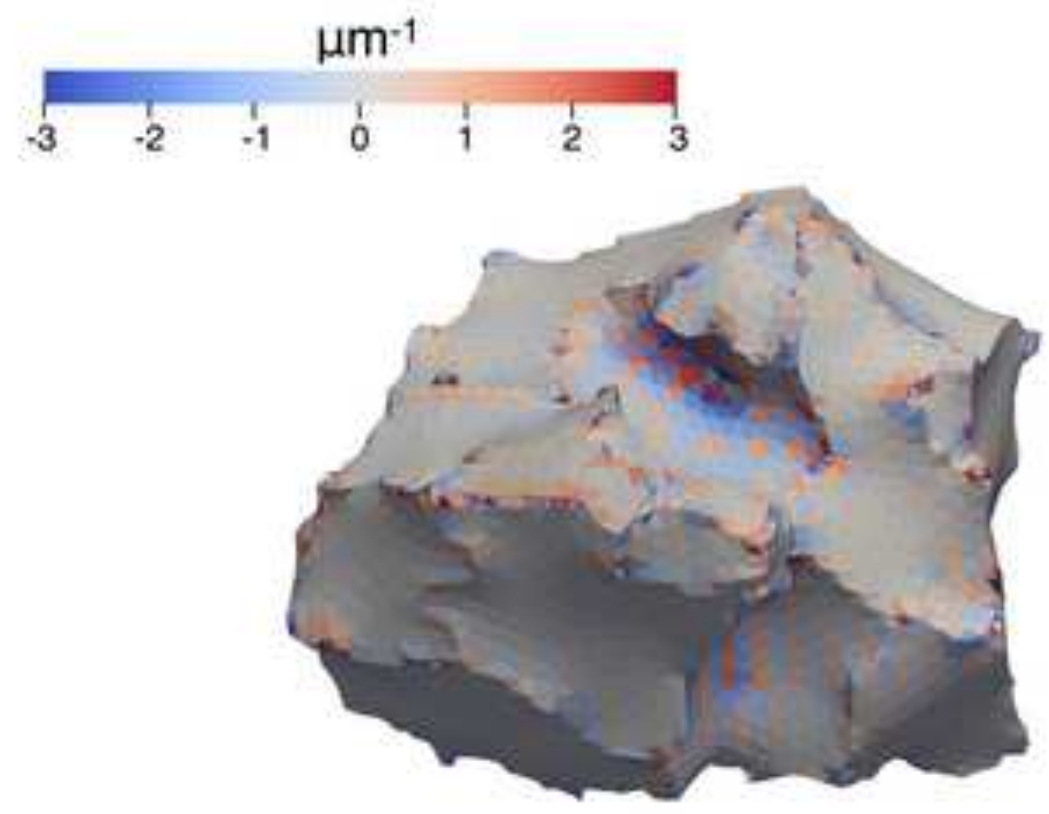

Grain Boundary, Colored by Mean Curvature

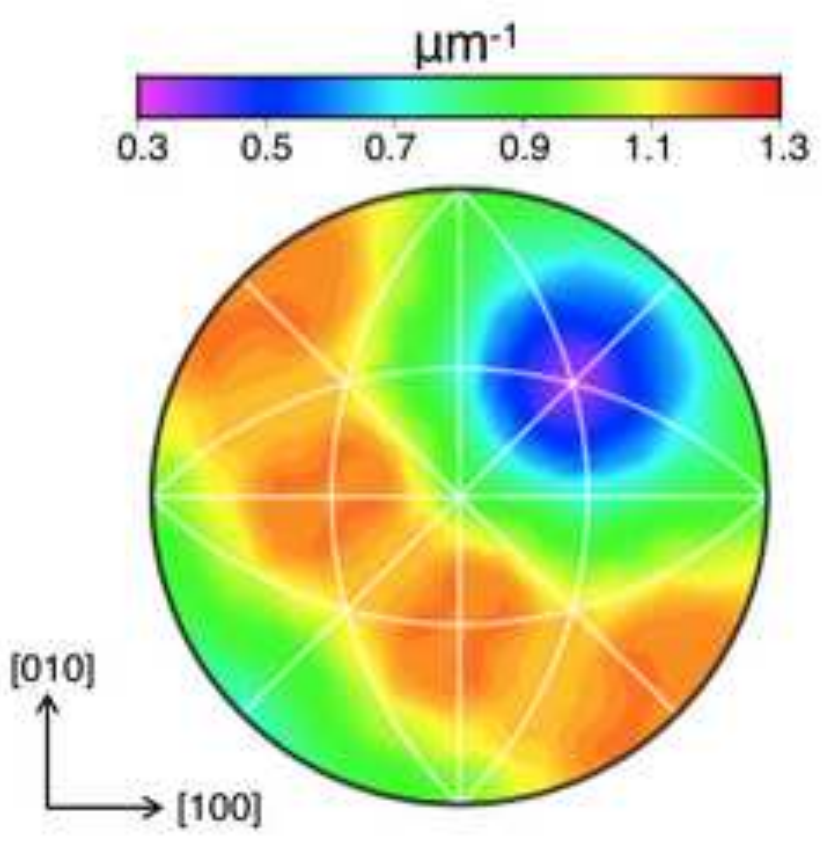

Mean Curvature of $\Sigma 3\left(60^{\circ} /[111]\right)$ Boundary 